Disclosure of Interests: None declared

DOI: 10.1136/annrheumdis-2021-eular.2245

\section{AB0126 METHOTREXATE MAINTENANCE THERAPY DURING THE TARGETED THERAPY ERA IN RHEUMATOID ARTHRITIS}

S. Abdellaoui ${ }^{1}$, S. Lefkir ${ }^{1} .{ }^{1}$ Issad Hassani Beni Messous Hospital, Rheumatology, Algiers, Algeria

Background: The persistence with methotrexate (MTX) at 1 year or 5 years in rheumatoid arthritis (RA) is very variable and intolerance remains the main cause of discontinuation of treatment. Changes in treatment with the strategy of adding "add-on" to a targeted therapy when the conventional DMARD becomes insufficient to optimally control the disease may decrease the maintenance MTX therapy rates, particularly for biologics with a Marketing Authorization as monotherapy. Objectives: The main objective of this study was to determine the rate of maintenance at 1 year of the combination therapy with different biologics, to compare the evaluation scores in patients receiving treatment as monotherapy (biological only) versus combination therapy with methotrexate and to analyze predictive factors for MTX maintenance therapy.

Methods: We performed a descriptive study of 56 patients with RA meeting the criteria of the ACR 2010. Statistical analysis SPSS.20 Software.

Results: These were 56 patients: 93\% women and 7\% men, mean age 46.74 years with an average duration of the disease of 14.74 years. $71.42 \%$ of patients were under corticosteroids taken for a period of 13 years with an average dose of $5.04 \mathrm{mg} /$ day. BMI was highin $23.80 \%$ of patients, $30.95 \%$ had at least one associated comorbidity. The RF was positive in $61.90 \%$ and ACPA positive in $78.57 \%$ of cases. $47.62 \%$ were under MTX taken for 6.55 years with a dose of $13.03 \mathrm{mg} /$ week on average: $68.75 \%$ by oral intake and $31.25 \%$ by subcutaneous intake with an average duration of setting before the switch of 8 years. 28 patients were on rituximab, 19 patients on tocilizumab and 9 patients on TNFi. 88.09\% did not havenot received from bDMARD before. The mean ESR was $36.57 \mathrm{~mm}$ $\mathrm{H} 1$ and CRP was $8.56 \mathrm{mg} / \mathrm{L}$. DAS28 at baseline was 6.81 and the current DAS 28 was 2.95 and the HAQ was 1.11 . The rate of MTX maintenance therapy at 1 year was $36.84 \%$, with rituximab, $64.28 \%$ with tocilizumab and $88 \%$ with TNFi. The comparison between the combination therapy with MTX and monotherapy groups showed a significant difference for the number of tender joints NTJ (3.5 vs 2.61), however, no significant difference concerning DAS28 value (2.98 vs 3.06), number of swollen joints NSJ (0.29 vs 0.22$)$ and HAQ (1.09 vs 1.15). Regarding predictive factors of MTX maintenance therapy: Significant correlation was found with a high DAS 28 at baseline, however no correlation concerning the positivity of ACPA, duration of the disease, NTJ, NSJ, ESR, HAQ and the high dose of MTX. Reduction in persistence has found a significant correlation with non-naive RA of $\geq 1$ bDMARD, disease activity score, type of biotherapy (antiTNF / rituximab vs Tocilizumab), young age, however no influence was found with a high $\mathrm{BMI}, \mathrm{HAQ}$, the presence of comorbidities or the withdrawal of corticosteroids. The increase in the persistence of combination therapy was associated with male gender and RF seropositivity only.

Conclusion: Drug persistence is an important aspect of treatment effectiveness. For rheumatologists, knowledge of the factors that predict whether to maintain the combination therapy with methotrexate, increase or reduce the persistence is of great interest when choosing a new treatment to initiate in patients with RA. REFERENCES:

[1] Lavielle.M and Dougados.M. Targeted therapies in rheumatoid arthritis:

Combination with conventional synthetic disease modifying antirheumatic drugs or monotherapy? Jone Bone Spine 2018;85:3-9.

Disclosure of Interests: None declared

DOI: 10.1136/annrheumdis-2021-eular.2346

\section{AB0127 1 COMBINATION THERAPY WITH METHOTREXATE AND QUALITY OF LIFE IN RHEUMATOID ARTHRITIS}

S. Abdellaoui ${ }^{1}$, S. Lefkir ${ }^{1} .{ }^{1}$ Issad Hassani Beni Messous Hospital, Rheumatology, Algiers, Algeria

Background: The level of quality of life in patients with rheumatoid arthritis (RA) is often overlooked. These patients suffer from an often-precarious quality of life resulting in pain, joint destruction and fatigue.
Objectives: The main objective of this study was to compare the level of quality of life in patients with RA receiving treatment with disease-modifying anti-rheumatic drugs (DMARDs): monotherapy (biological only) versus combination therapy with methotrexate.

Methods: We performed a descriptive study of 56 patients with RA meeting the criteria of the ACR 2010. The SPSS.20 software was used for statistical analyses. Results: These were 56 patients: 93\% women and 7\% men, mean age 46.74 years with an average duration of the disease of 14.74 years. 28 patients were on rituximab, 19 patients on tocilizumab and 9 patients on TNFi. The mean HAQ was $1.11 .52 .38 \%$ of patients were on biological alone as monotherapy. The Compliance Questionnaire Rheumatology score (CQR19) was 55.15 / 100, the RAID (Rheumatoid Arthritis Impact of Disease) score was 3.08 / 10, the SF36 of 56.01 / 100, the AIMS score of 2.10 / 10 for social activity, 2.44 / 10 for pain, 3.32 / 10 for depression and 4.06 for physical activity.

The comparison between the 2 groups (Combination therapy vs monotherapy) did not find any significant difference in terms of quality of life parameters: An SF-36 score $>55$ was found in $56 \%$ in patients on combination therapy vs. $44 \%$ on monotherapy, the RAID score was 3.02 vs. 3.12 , AIMS social activity 2.08 vs 2.13 , AIMS pain 2.33 vs 2.59 , AIMS activity physics of 4 vs 4.11

Conclusion: Our study did not demonstrate any superiority of the combination with methotrexate in improving quality of life. The use of biotherapy in patients with RA has been shown to be an important pharmacological strategy for the overall management of the disease.

\section{REFERENCES:}

[1] Lavielle.M and Dougados.M. Targeted therapies in rheumatoid arthritis: Combination with conventional synthetic disease modifying antirheumatic drugs or monotherapy? Jone Bone Spine 2018; 85:3-9.

Disclosure of Interests: None declared

DOI: 10.1136/annrheumdis-2021-eular.2453

\section{AB0128 1 CONSTRUCTION OF THE VETERANS AFFAIRS NATIONAL RHEUMATOID ARTHRITIS DATABASE (VANRAD)}

A. Joseph ${ }^{1,2}$, J. Yanagida ${ }^{2}$, X. Huang ${ }^{1,2}$, P. Ranganathan ${ }^{1,2}$, M. Laurie ${ }^{3}$, H. Xian ${ }^{2,4}$, S. Eisen ${ }^{1,2}$. ${ }^{1}$ Washington University School of Medicine, Rheumatology, Saint Louis, Missouri, United States of America; ${ }^{2}$ VA St. Louis Health Care System, Rheumatology, Saint Louis, Missouri, United States of America; ${ }^{3}$ Bristol Myers Squibb, Health Economics \& Outcomes Research, Lawrence Township, United States of America; ${ }^{4}$ Saint Louis University College for Public Health \& Social Justice, Epidemiology \& Biostatics, Saint Louis, Missouri, United States of America

Background: The Department of Veterans Affairs (DVA) provides comprehensive medical care at minimal or no cost to 9 million veterans annually through 170 medical centers and 1074 outpatient clinics across the United States. In 1999, the DVA established a national, fully integrated electronic health record (EHR), which now includes approximately 24 million veterans. However, few studies have used VA EHR data to examine the validity of diagnoses of rheumatoid arthritis (RA). Objectives: Develop a validated, national database (VANRAD) of patients with RA who received VA care since International Classification of Diseases, tenth revision (ICD-10) coding was introduced in 2015. The database will provide infrastructure for retrospective and prospective research to address the 'real-world' care of patients with RA.

Methods: Patients with the following criteria were identified from the VA EHR as of October 2, 2020: (a) $\geq 1$ ICD-10 diagnosis code of RA; (b) treatment with $\geq 1$ disease-modifying anti-rheumatic drug (DMARD); (c) $\geq 2$ VA rheumatology clinic visits; and $(d) \geq 1$ rheumatoid factor (RF) or anti-cyclic citrullinated peptide (aCCP) antibody test result. From this group, 553 EHRs were randomly selected for review. The 'gold standard' for the diagnosis of RA was the treating rheumatologist's diagnosis, documented in the EHR.

Results: 27,482 patients met eligibility criteria. Sociodemographic characteristics were: $85.6 \%$ male, mean age of 69.7 years $(y)(S D=10.9$ y; range $=21.9$ y to $100.5 \mathrm{y}), 76.4 \%$ white, $17.0 \%$ African American; and mean VA care duration 14.1 y $(S D=5.3 y$, range $=0.04$ y to $20.0 \mathrm{y})$.

For patients with $\geq 1 \mathrm{RF}$ or aCCP test, the positive predictive value (PPV) for RA ranged from $65.3 \%$ (aCCP-/RF-) to $95.8 \%$ (aCCP+/RF+); rheumatologists' likelihood of a 'possible' diagnosis was higher if the aCCP test result was negative or not available (Table 1). Excluding patients with a second rheumatologic diagnosis did not improve PPV results (data not shown). 